\title{
Lepton Flavor Violation Induced by a Neutral Scalar at Future Lepton Colliders
}

\author{
P. S. Bhupal Dev, ${ }^{1}$ Rabindra N. Mohapatra, ${ }^{2}$ and Yongchao Zhang ${ }^{1}$ \\ ${ }^{1}$ Department of Physics and McDonnell Center for the Space Sciences, Washington University, St. Louis, Missouri 63130, USA \\ ${ }^{2}$ Maryland Center for Fundamental Physics, Department of Physics, University of Maryland, College Park, Maryland 20742, USA
}

(Received 7 December 2017; published 31 May 2018)

\begin{abstract}
Many new physics scenarios beyond standard model often necessitate the existence of a (light) neutral scalar $H$, which might couple to the charged leptons in a flavor violating way, while evading all existing constraints. We show that such scalars could be effectively produced at future lepton colliders, either on shell or off shell depending on their mass, and induce lepton flavor violating (LFV) signals, i.e., $e^{+} e^{-} \rightarrow$ $\ell_{\alpha}^{ \pm} \ell_{\beta}^{\mp}(+H)$ with $\alpha \neq \beta$. We find that a large parameter space of the scalar mass and the LFV couplings can be probed well beyond the current low-energy constraints in the lepton sector. In particular, a scalar-loop induced explanation of the long-standing muon $g-2$ anomaly can be directly tested in the on-shell mode.
\end{abstract}

DOI: 10.1103/PhysRevLett.120.221804

Introduction.-The observation of neutrino oscillations [1] suggests that the lepton family numbers are violated. It also calls for an extension of the standard model (SM) to include neutrino mass terms, which necessarily induce charged lepton flavor violation (cLFV). In the minimal extension of the SM with Dirac neutrinos, cLFV rates are highly suppressed due to small neutrino masses (compared to the electroweak scale). This makes the experimental searches for cLFV all the more interesting, because any observable effect must come from physics beyond the minimally extended SM related to the origin of neutrino mass.

There are various theoretical models of new physics which lead to cLFV effects at an observable level $[2,3]$. They generally involve extending the Higgs sector, which allows flavor-violating Yukawa couplings of new neutral scalars beyond the SM. In particular, if any of the new neutral scalars (call it $H$ ) is (almost) hadrophobic, it could remain sufficiently light and contribute sizably to cLFV, while easily evading the direct searches at hadron colliders, as well as the low-energy quark flavor constraints, such as the rare flavor-changing decays and oscillations of $K$ and $B$ mesons. Some well-motivated examples include supersymmetric models with leptonic $R$-parity violation [4], left-right symmetric models [5], mirror models [6], and two-Higgs doublet models [7], where the cLFV coupling might arise at tree or loop level [8].

In this Letter, we show that such scenarios of neutral scalar-induced cLFV can be effectively probed in a model-

Published by the American Physical Society under the terms of the Creative Commons Attribution 4.0 International license. Further distribution of this work must maintain attribution to the author(s) and the published article's title, journal citation, and DOI. Funded by SCOAP ${ }^{3}$. independent way at future lepton colliders, such as the Circular Electron-Positron Collider (CEPC) [15], International Linear Collider (ILC) [16], Future Circular Collider (FCC-ee) [17], and Compact Linear Collider (CLIC) [18]. Compared to the hadron colliders, the lepton colliders are generally very "clean" and the SM processes therein are well understood, which render them primary facilities to search for new physics via the cLFV signals $e^{+} e^{-} \rightarrow \ell_{\alpha}^{ \pm} \ell_{\beta}^{\mp}+X$ (with $\alpha, \beta=e, \mu, \tau$ and $\alpha \neq \beta$ ). Previous studies of LFV at lepton colliders have either been performed in the framework of effective four-fermion couplings $[19,20]$ or in the context of flavor-violating SM Higgs decays [21] and tau decays [22] or with doubly charged scalars [23]. Here we include both on- and off-shell production of the new neutral scalar $H$ (including resonance) at lepton colliders, which enables us to derive the LFV sensitivity as a function of the mass $m_{H}$ for a direct comparison with the current bounds from low-energy experiments. Moreover, for $m_{H}$ small compared to the center-of-mass energy, the effective theory approximation does not work.

Without loss of generality, we can write the effective Yukawa couplings of $H$ to the charged leptons as

$$
\mathcal{L}_{Y}=h_{\alpha \beta} \bar{\ell}_{\alpha, L} H \ell_{\beta, R}+\text { H.c. }
$$

Here, for simplicity we have assumed the couplings are all real and chirality independent and thus symmetric. The scalar $H$ may or may not be responsible for symmetry breaking and/or mass generation of other particles in realistic models, where it could be part of a singlet, doublet, or triplet scalar field. We assume that it is $C P$ even and its mixing with and/or coupling to the SM Higgs boson is small. If the scalar is $C P$ odd, the limits and prospects derived in this Letter would not change significantly. Even though there are all varieties of stringent low-energy cLFV 


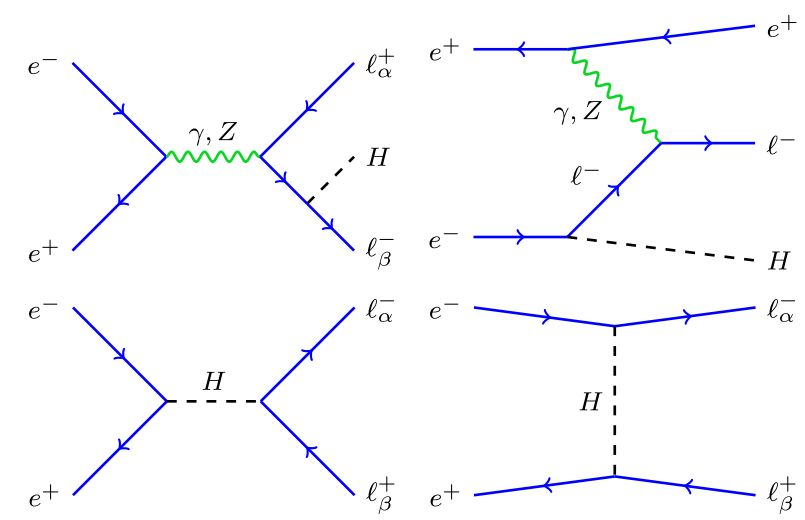

FIG. 1. Examples of LFV signal from on-shell, off-shell, and resonant neutral scalar production at $e^{+} e^{-}$collider.

constraints, such as $\ell_{\alpha} \rightarrow \ell_{\beta} \gamma, \ell_{\alpha} \rightarrow 3 \ell_{\beta}, 2 \ell_{\beta} \ell_{\gamma}$ [1], only a few of them are directly relevant to the LFV prospects discussed below. With an $\mathrm{ab}^{-1}$ level of integrated luminosity, a large parameter space of $m_{H}$ and $h_{\alpha \beta}$ could be probed, well beyond the current cLFV constraints and complementary to the projected low-energy constraints from future experiments at the intensity frontier [24]. In addition, the Lagrangian (1) also gives rise to a one-loop contribution to the lepton anomalous magnetic moment. In particular, the long-standing muon $g-2$ discrepancy [1] could also be tested directly at lepton colliders.

On-shell LFV.-If kinematically allowed, the neutral scalar $H$ can be directly produced at lepton colliders, in association with a pair of flavor-changing leptons through the couplings in Eq. (1), i.e., $e^{+} e^{-} \rightarrow \ell_{\alpha}^{ \pm} \ell_{\beta}^{\mp} H$ (with $\alpha \neq \beta$ ), as shown in Fig. 1 (top panel).

$e-\mu$ coupling: Here, for simplicity, we assume the other two $h_{e \tau}$ and $h_{\mu \tau}$ are vanishing. It should be emphasized that the amplitudes in Fig. 1 depend only on the LFV couplings $h_{\alpha \beta}$ (here, $\alpha \beta=e \mu$ ), and thus could be easily made to satisfy the rare lepton decay constraints, such as $\mu \rightarrow$ eee and $\mu \rightarrow e \gamma$, which depend on the product $\left|h_{e e}^{\dagger} h_{e \mu}\right|$. Similarly, with vanishing or suppressed couplings to the quark sector, the $\mu-e$ conversion limits are irrelevant. Finally, for real Yukawa couplings, we do not either have any limits from electric dipole moment. Thus, we are left only with the following constraints (summarized in Table I):

(i) Muonium-antimuonium oscillation: This could occur in both $s$ and $t$ channels [8]. The oscillation probability $\mathcal{P} \propto\left|h_{e \mu}\right|^{4} / m_{H}^{4}$. The MACS experiment [25] could then exclude a large parameter space, as shown in the left panel of Fig. 3.

(ii) $(g-2)_{e}$ : The anomalous magnetic moment of electron $a_{e}$ receives a contribution from the $H-\mu$ loop [8]. As a result of the precise measurement of $a_{e}$ [26], the constraint on $h_{e \mu}$ is comparable to that from muonium oscillation, as shown in the left panel of Fig. 3. To explain the long-standing theoretical and experimental discrepancy
TABLE I. Current experimental constraints on the LFV couplings. The $\Delta a_{e, \mu}$ constraints have an additional logarithmic dependence on the scalar mass [8].

\begin{tabular}{lr}
\hline \hline Process & Constraints $\times\left(\mathrm{GeV} / m_{H}\right)^{2}$ \\
\hline Muonium oscillation & $\left|h_{e \mu}\right|^{2}<1.0 \times 10^{-7}$ \\
$\Delta a_{e}$ & $\left|h_{e \mu}\right|^{2}<6.2 \times 10^{-8}$ \\
$\Delta a_{\mu}$ & $\left|h_{e \tau}\right|^{2}<6.9 \times 10^{-9}$ \\
$e e \rightarrow \mu \mu$ & $\left|h_{\mu \tau}\right|^{2}<4.4 \times 10^{-7}$ \\
$e e \rightarrow \tau \tau$ & $\left|h_{e \mu}\right|^{2}<1.6 \times 10^{-7}$ \\
$\mu^{-} \rightarrow e^{-} e^{+} e^{-}$ & $\left|h_{e \tau}\right|^{2}<1.0 \times 10^{-7}$ \\
$\tau^{-} \rightarrow e^{-} e^{+} e^{-}$ & $\left|h_{e e}^{\dagger} h_{e \mu}\right|<6.6 \times 10^{-11}$ \\
$\tau^{-} \rightarrow \mu^{-} e^{+} e^{-}$ & $\left|h_{e e}^{\dagger} h_{e \tau}\right|<2.6 \times 10^{-8}$ \\
$\tau^{-} \rightarrow \mu^{+} e^{-} e^{-}$ & $\left|h_{e e}^{\dagger} h_{\mu \tau}\right|<1.5 \times 10^{-8}$ \\
$\tau^{-} \rightarrow e^{-} \gamma$ & $\left|h_{e \mu}^{\dagger} h_{e \tau}\right|<1.9 \times 10^{-8}$ \\
$\tau^{-} \rightarrow \mu^{-} \gamma$ & $\left|h_{e e}^{\dagger} h_{e \tau}\right|<1.0 \times 10^{-6}$ \\
$\Delta a_{e}$ & $\left|h_{e \mu}^{\dagger} h_{e \tau}\right|<1.2 \times 10^{-6}$ \\
$e e \rightarrow e e, \tau \tau$ & $\left|h_{e}^{\dagger} h_{e \tau}\right|<1.1 \times 10^{-7}$ \\
$e e \rightarrow \mu \mu, \tau \tau$ & $\left|h_{e \mu}^{\dagger} h_{e \tau}\right|<1.0 \times 10^{-8}$ \\
\hline \hline
\end{tabular}

of the muon $g-2$, i.e., $\Delta a_{\mu}=(2.87 \pm 0.80) \times 10^{-9}$ [1], the LFV coupling $h_{e \mu}$ is required to be larger, as shown in the left panel of Fig. 3 (by the brown line and the green, yellow bands corresponding, respectively, to the central value and the $1 \sigma, 2 \sigma$ ranges), which is already excluded by the $(g-2)_{e}$ data. See, however, the $\mu \tau$ sector in the right panel of Fig. 3 for an explanation of $(g-2)_{\mu}$ in this setup.

(iii) $e^{+} e^{-} \rightarrow \mu^{+} \mu^{-}$: A $t$-channel $H$ could mediate the scattering $e^{+} e^{-} \rightarrow \mu^{+} \mu^{-}$, which interferes with the SM diagrams in the $s$ channel [27]. Both the total cross section and differential distributions would be modified by the presence of $H$, depending on its mass and the coupling $h_{e \mu}$. In the heavy $H$ limit, the LEP data exclude an effective

TABLE II. Benchmark configurations of future lepton colliders CEPC and ILC and the expected total cross sections of the onshell and off-shell production of $H$, up to the LFV couplings squared, in the light $H$ limit. The values in parentheses are for $m_{H}=100 \mathrm{GeV}$.

\begin{tabular}{lcc}
\hline \hline Collider & CEPC & ILC \\
\hline$\sqrt{s}$ & $240 \mathrm{GeV}$ & $1 \mathrm{TeV}$ \\
Luminosity & $5 \mathrm{ab}^{-1}$ & $1 \mathrm{ab}^{-1}$ \\
Cuts & $p_{T}(\ell)>10 \mathrm{GeV},|\eta(\ell)|<2.5$ \\
$\sigma\left(e \mu+H_{3}\right) /\left|h_{e \mu}\right|^{2}$ & $8.9 \times 10^{4}(390) \mathrm{fb}$ & $1.1 \times 10^{5}(2800) \mathrm{fb}$ \\
$\sigma\left(e \tau+H_{3}\right) /\left|h_{e \tau}\right|^{2}$ & $5.3 \times 10^{4}(650) \mathrm{fb}$ & $6.6 \times 10^{4}(1700) \mathrm{fb}$ \\
$\sigma\left(\mu \tau+H_{3}\right) /\left|h_{\mu \tau}\right|^{2}$ & $2100(5.0) \mathrm{fb}$ & $5700(3.5) \mathrm{fb}$ \\
$\sigma(e \tau) /\left|h_{e e}^{\dagger} h_{e \tau}\right|^{2}$ & $4.8 \times 10^{5} \mathrm{fb}$ & $2.8 \times 10^{4} \mathrm{fb}$ \\
$\sigma(\mu \tau) /\left|h_{e e}^{\dagger} h_{\mu \tau}\right|^{2}$ & $1.6 \times 10^{5} \mathrm{fb}$ & $9300 \mathrm{fb}$ \\
$\sigma(\mu \tau) /\left|h_{e \mu}^{\dagger} h_{e \tau}\right|^{2}$ & $1.6 \times 10^{5} \mathrm{fb}$ & $9300 \mathrm{fb}$ \\
\hline \hline
\end{tabular}



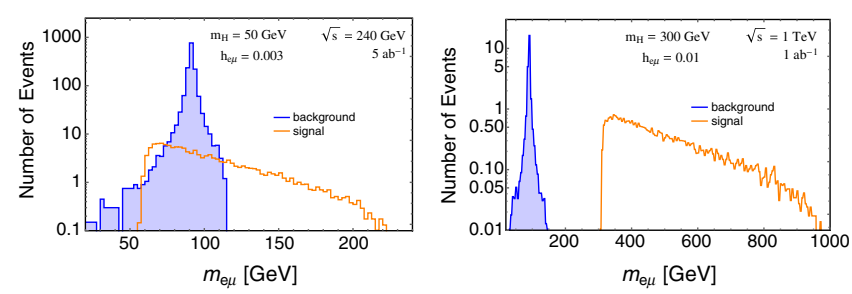

FIG. 2. $m_{e \mu}$ invariant mass distributions for the SM background $e^{+} e^{-} \rightarrow Z h$ and the signal $e^{+} e^{-} \rightarrow e^{ \pm} \mu^{\mp} H$ at CEPC (left) and ILC (right).

cutoff scale $\Lambda \simeq m_{H} / h_{e \mu}$ [28]. When $H$ is lighter than the center-of-mass energy $\sqrt{s}$, the limits on $\Lambda$ do not apply, and we consider the $H$ propagator: $\left(q^{2}-m_{H}^{2}\right)^{-1}=$ $\left(-s \cos \theta / 2-m_{H}^{2}\right)^{-1}$. For simplicity we take an average over the scattering angle $\langle\cos \theta\rangle \simeq 1 / 2$ to interpret the LEP constraints. Then, in the limit of $m_{H} \ll \sqrt{s}$, the propagator is dominated by the $q^{2}$ term, and the $e e \rightarrow \mu \mu$ limit in Fig. 3 approaches a constant, as expected.

To be specific, we consider two benchmark configurations for future lepton colliders: i.e., the CEPC [15] and ILC [16], with the center-of-mass energies $\sqrt{s}$, integrated luminosities, and the nominal cuts on the leptons $\ell$ (implemented by using CalcHEP [29]) summarized in Table II. The total cross sections $\sigma\left[e e \rightarrow \ell_{\alpha} \ell_{\beta}(+H)\right]$ in the light $H$ limit (and $m_{H}=100 \mathrm{GeV}$ for the on-shell production) are also presented in the Table II, with a conservative efficiency of $60 \%$ for the $\tau$ lepton [16]. The systematic uncertainties such as initial state radiation, bremmstrahlung, and the electron and muon efficiencies lead only up to a few percent correction to the total cross sections $[15,16]$.

The SM background is dominated by particle misidentification from the Higgsstrahlung process $e^{+} e^{-} \rightarrow Z h$ with one of the $e(\mu)$ from $Z$ decay misidentified as $\mu$ (e) [30] (see also Ref. [31]). The invariant mass $m_{e \mu}$ distributions from the on-shell production of $e \mu H$ can be easily distinguished from the backgrounds, as exemplified in Fig. 2, with $m_{H}=50 \mathrm{GeV}$ and $h_{e \mu}=0.003$ at CEPC, and with $m_{H}=300 \mathrm{GeV}$ and $h_{e \mu}=0.01$ at ILC. Removing the $Z$-resonance peak, the LFV signal is almost background free. Summing all the bins off the $Z$ peak, the signal $(S)$ to background $(B)$ significance $S / \sqrt{S+B}$ for the examples in Fig. 2 are, respectively, 55 and 61.

After being produced, $H$ could decay back into the charged lepton pairs or other SM particles. Reconstructing the $H$ peak from the decay products could improve further the significance of the LFV signals, which are, however, rather model dependent. To work in a model-independent way, we consider three benchmark values, where $1 \%, 10 \%$, or $100 \%$ of the decay products of $H$ are visible and can be reconstructed. The corresponding LFV prospects are shown in the left panel of Fig. 3, where we have assumed a minimum of 10 signal events at both CEPC and ILC. It is clear from Fig. 3 that with a BR of $\gtrsim 10 \%$, a large region of $m_{H}$ and $\left|h_{e \mu}\right|$ can be probed in future lepton colliders, which extends the limits well beyond what is currently available.

$e-\tau$ coupling: Turning now to the coupling $h_{e \tau}$, the most stringent limit comes from the electron $g-2$, which is similar to the case of $h_{e \mu}$ except for the enhancement by the $\tau$ mass [cf. Eq. (S13) in the Supplemental Material [8]], as shown by the pink region in the middle panel of Fig. 3. The LEP $e^{+} e^{-} \rightarrow \tau^{+} \tau^{-}$limit is slightly stronger than the muon case [28], as shown by the shaded purple region in Fig. 3. The reconstruction of the $\tau$ lepton is more challenging than $\mu$, and thus the prospects of $h_{e \tau}$ are somewhat weaker than $h_{e \mu}$, but there is still ample parameter space to probe at both CEPC and ILC, as long as the effective BR is $\gtrsim 10 \%$.

$\mu-\tau$ coupling: Turning now to the coupling $h_{\mu \tau}$, there are currently no experimental limits, except for the muon $g-2$ discrepancy. This could be explained in the presence of $H$ when it couples to muon and tau, as shown by the brown line and the green and yellow bands in the right panel of Fig. 3, while the shaded region is excluded by the current muon $g-2$ data at the $5 \sigma$ level. As $\mu \tau$ can only be produced in the $e^{+} e^{-}$collider in the $s$ channel in Fig. 1, the
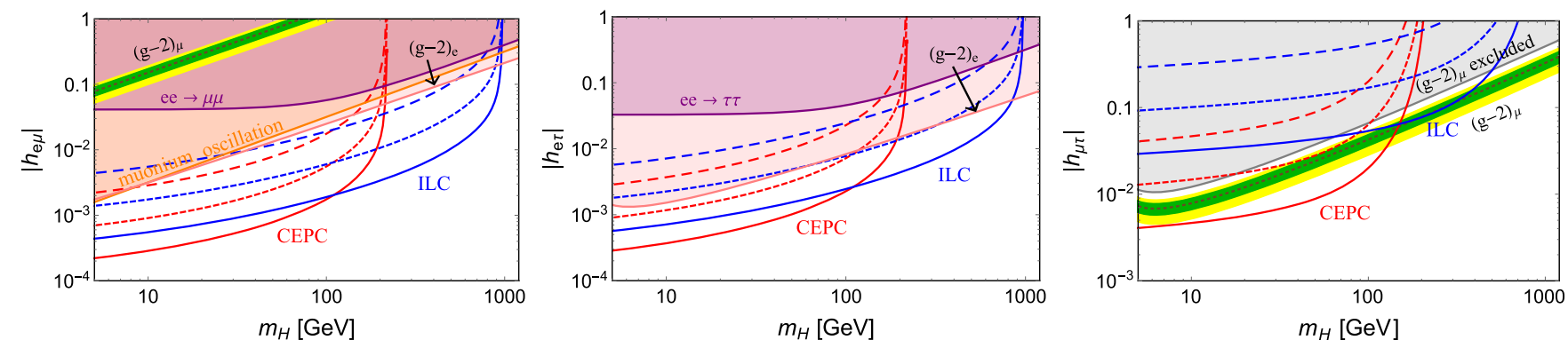

FIG. 3. Prospects of probing LFV couplings $h_{\alpha \beta}(\alpha \neq \beta)$ from searches of $e^{+} e^{-} \rightarrow \ell_{\alpha}^{ \pm} \ell_{\beta}^{\mp} H$ at CEPC (red, $\sqrt{s}=240 \mathrm{GeV}$ and $\mathcal{L}=5 \mathrm{ab}^{-1}$ ) and ILC (blue, $1 \mathrm{TeV}$ and $1 \mathrm{ab}^{-1}$ ). Here we have assumed $10 \mathrm{LFV}$ signal events and a BR of $1 \%$ (long-dashed), $10 \%$ (shortdashed), or $100 \%$ (solid) from $H$ decay to be visible. In the left panel, the region shaded in orange, pink, and purple and, respectively, excluded by muonium oscillation, $(g-2)_{e}$ and $e e \rightarrow \mu \mu$ data; in the middle panel, the pink and purple regions are excluded by $(g-2)_{e}$ and $e e \rightarrow \tau \tau$ data; in the right panel the gray region is disfavored by the $(g-2)_{\mu}$ data at the $5 \sigma$ confidence level. In the left and right panels, the brown line could fit the central value of $\Delta a_{\mu}$, and the green and yellow bands cover the $1 \sigma$ and $2 \sigma$ ranges of $\Delta a_{\mu}$. 
production cross section is smaller than those of $e \mu$ and $e \tau$. From Eq. (S13) in the Supplemental Material [8] (with the couplings and lepton masses changed accordingly), the $(g-2)_{\mu}$ anomaly can be directly tested at CEPC up to a scalar mass of $\simeq 100 \mathrm{GeV}$, as shown in Fig. 3, as long as there is a sizable BR of $H$ into visible states. With a larger luminosity being planned [17], FCC-ee could do even better.

Off-shell (and resonant) LFV.-The LFV signals could also be produced from an off-shell $H$, i.e., $e^{+} e^{-} \rightarrow \ell_{\alpha}^{ \pm} \ell_{\beta}^{\mp}$, as shown in Fig. 1 (bottom panel). This could occur in both the $s$ and $t$ channels; in the $s$ channel $H$ is on shell if the colliding energy $\sqrt{s} \simeq m_{H}$ (resonance). Different from the on-shell case, the off-shell production amplitudes have a quadratic dependence on the Yukawa couplings (some of them might be flavor conserving), and thus largely complementary to the on-shell LFV searches.

The amplitude $e^{+} e^{-} \rightarrow e^{ \pm} \mu^{\mp}$ is proportional to $h_{e e}^{\dagger} h_{e \mu}$. This is tightly constrained by the $\mu \rightarrow e e e$ data in Table I, leaving no hope to see any signal in this channel. In the $\tau$ lepton sector, the LFV decay constraints are comparatively much weaker. In the parameter space of interest $m_{H} \gtrsim m_{\tau}$, the limits on $\left|h^{\dagger} h\right| / m_{H}^{2}$ are almost constants, as in effective field theories with superheavy mediators. These constraints are all presented in Fig. 4, with the shaded regions excluded. The analytic formulas and calculation details are given in Ref. [8]. As for the on-shell case above, the couplings $h_{e \beta}(\beta=e, \mu, \tau)$ are constrained, respectively, by the LEP $e^{+} e^{-} \rightarrow \ell^{+} \ell^{-}$data. Thus, we can set upper limits on the couplings $\left|h_{e e}^{\dagger} h_{e \tau}\right|$ and $\left|h_{e \mu}^{\dagger} h_{e \tau}\right|$, as shown in Table I and the left and right panels of Fig. 4, which get weaker for lighter $H$, as in the on-shell case.

Given $h_{e e}$ and $h_{e \tau}$, the electron $g-2$ receives both contributions from the $H$ loops with an $e / \tau$ in the intermediate state, and for a fixed value of $\left|h_{e e}^{\dagger} h_{e \tau}\right|$, the weakest $(g-2)_{e}$ constraint occurs when $h_{e e} m_{e} \sim h_{e \tau} m_{\tau}$, with the two loops contributing almost equally. Similarly, one can obtain the $(g-2)_{e}$ limit on $\left|h_{e \mu}^{\dagger} h_{e \tau}\right|$, which induces the $\mu / \tau$-mediated diagrams. Both the constraints are presented in the left and right panels of Fig. 4. Note that the muon $g-2$ cannot be used to set unambiguous limits on the combinations $\left|h_{e e}^{\dagger} h_{\mu \tau}\right|$ and $\left|h_{e \mu}^{\dagger} h_{e \tau}\right|$, although the couplings $h_{\mu \tau}$ and $h_{e \mu}$ could contribute to $(g-2)_{\mu}$ by themselves.

The dominant SM backgrounds are from the process $e^{+} e^{-} \rightarrow W^{+} W^{-} \rightarrow e^{-} \tau^{+} \bar{\nu}_{e} \nu_{\tau}$, which is expected to be small, if we require the two charged leptons to be back to back and their reconstructed energy $E_{\ell} \simeq \sqrt{s} / 2$ [19]. The angular distributions of charged leptons can also be used to suppress the SM $W W$ backgrounds [32]. Assuming 10 signal events as above, the coupling $\left|h_{e e}^{\dagger} h_{e \tau}\right|$ could be probed up to $6.5 \times 10^{-5}\left(6.0 \times 10^{-4}\right)$ at CEPC (ILC) in the light $H$ limit, as shown in Fig. 4. At the resonance $m_{H} \simeq \sqrt{s}$, the production cross section can be greatly enhanced by $m_{H}^{2} / \Gamma_{H}^{2}$. To be specific, we have set the width $\Gamma_{H}=10$ (30) GeV at $\sqrt{s}=240 \mathrm{GeV}(1 \mathrm{TeV})$, where the prospects could be strengthened by roughly 1 order of magnitude (the dips in Fig. 4). For $m_{H}>\sqrt{s}$, the production rate diminishes rapidly as $H$ becomes heavier. An offshell $H$ could, however, be probed up to a few-TeV range, as shown in Fig. 4, and ILC is expected to be more promising than CEPC in this mass range, as a result of the higher $\sqrt{s}$.

The process $e^{+} e^{-} \rightarrow \mu^{ \pm} \tau^{\mp}$ could proceed via both the $s$ and $t$ channels, which depend on different couplings, namely, $\left|h_{e e}^{\dagger} h_{\mu \tau}\right|$ and $\left|h_{e \mu}^{\dagger} h_{e \tau}\right|$, and are constrained, respectively, by the rare decays $\tau^{-} \rightarrow \mu^{-} e^{+} e^{-}$and $\tau^{-} \rightarrow \mu^{+} e^{-} e^{-}$. Analogous to the $e \tau$ case above, a broad range of $m_{H}$ and $\left|h_{e e}^{\dagger} h_{\mu \tau}\right|$ could be probed in the $s$ channel, in particular in vicinity of the resonance, as shown by the middle panel of Fig. 4. In the $t$ channel, the cross sections are comparatively smaller, and the detectable regions are much narrower, as shown by the right panel of Fig. 4.

The future reaches of the LFV couplings in both the on-shell and off-shell production modes are collected in Table III. It is clear that orders of magnitude of the couplings can be probed at future lepton colliders, i.e., from $\sim 10^{-4}$ up to $\mathcal{O}(0.1)$ for a scalar mass range of $\sim \mathrm{GeV}$ to $200 \mathrm{GeV}$ at CEPC (900 at ILC) in the on-shell channel, and couplings from $\sim 10^{-4}$ up to $\mathcal{O}(1)$ for a mass range from $\sim 100 \mathrm{GeV}$ to few $\mathrm{TeV}$ in the offshell mode.
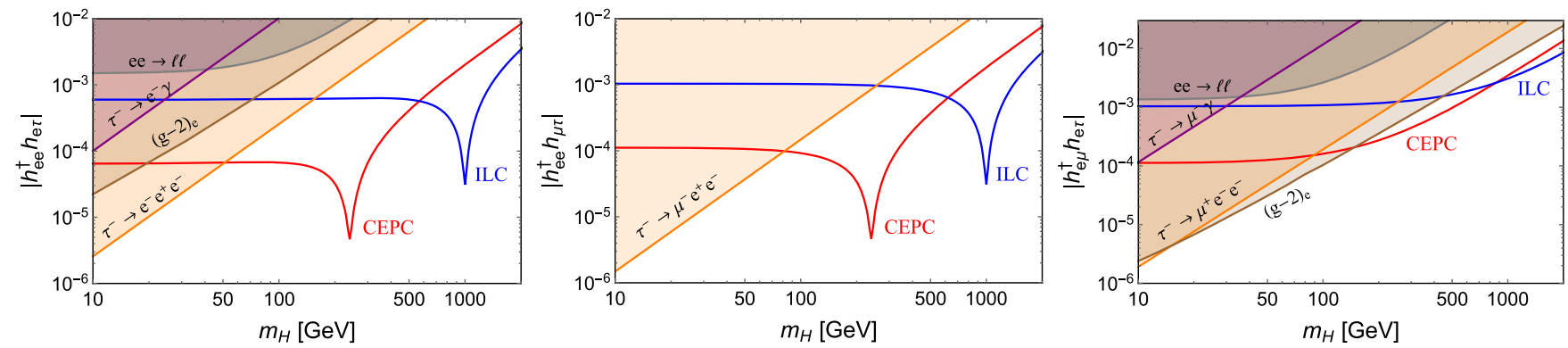

FIG. 4. Prospects of $\left|h_{e e}^{\dagger} h_{e \tau}\right|$ (left), $\left|h_{e e}^{\dagger} h_{\mu \tau}\right|$ (middle) and $\left|h_{e \mu}^{\dagger} h_{e \tau}\right|$ (right) from searches of $e^{+} e^{-} \rightarrow e^{ \pm} \tau^{\mp}, \mu^{ \pm} \tau^{\mp}$ at CEPC (red, $\sqrt{s}=240 \mathrm{GeV}, \mathcal{L}=5 \mathrm{ab}^{-1}$ ) and ILC (blue, $1 \mathrm{TeV}$ and $1 \mathrm{ab}^{-1}$ ). Here we have assumed 10 signal events. Also shown are the constraints from the rare lepton decays, $(g-2)_{e}$, and $e^{+} e^{-} \rightarrow \ell^{+} \ell^{-}$LEP data (cf. Table I). 
TABLE III. Reaches of the LFV couplings at future lepton colliders CEPC and ILC in both the on-shell and off-shell channel, with the BR of $1 \%, 10 \%$, and $100 \%$ for $H$ decay reconstructible in the on-shell channel.

\begin{tabular}{lcccc}
\hline \hline Collider & BR & $\left|h_{e \mu}\right|$ & $\left|h_{e \tau}\right|$ & $\left|h_{\mu \tau}\right|$ \\
\hline \multirow{6}{*}{ CEPC } & $1 \%$ & {$[0.0026,0.034]$} & $\ldots$ & $\ldots$ \\
& $10 \%$ & {$[0.0099,0.12]$} & {$[0.0009,0.0096]$} & {$[0.017,0.068]$} \\
& $100 \%$ & {$[0.00022,0.050]$} & {$[0.00029,0.015]$} & {$[0.0041,0.10]$} \\
ILC & $1 \%$ & {$[0.0099,0.12]$} & $\ldots$ & $\ldots$ \\
& $10 \%$ & {$[0.0014,0.047]$} & {$[0.0056,0.023]$} & $\ldots$ \\
& $100 \%$ & {$[0.00044,0.050]$} & {$[0.00057,0.054]$} & {$[0.046,0.27]$} \\
\hline Collider & $\left|h_{e e}^{\dagger} h_{e \tau}\right|$ & $\left|h_{e e}^{\dagger} h_{\mu \tau}\right|$ & $\left|h_{e \mu}^{\dagger} h_{e \tau}\right|$ \\
\hline CEPC & $>6.5 \times 10^{-5}$ & $>1.1 \times 10^{-4}$ & $>2.0 \times 10^{-4}$ \\
ILC & $>6.0 \times 10^{-4}$ & $>1.0 \times 10^{-3}$ & $>1.5 \times 10^{-3}$ \\
\hline \hline
\end{tabular}

Conclusion.-We have shown that a hadrophobic neutral scalar $H$, which is well motivated in a large class of new physics scenarios, can be probed in an $e^{+} e^{-}$collider via its LFV couplings to the charged lepton sector. We present a model-independent analysis of how far the LFV coupling strengths and the scalar mass can be probed beyond the existing limits from the low-energy sector. In particular, we find that the full mass and coupling range of the scalar, that can explain the muon $g-2$ anomaly, can be tested in the future lepton colliders. This is largely complementary to the searches of LFV in the low-energy experiments and hadron colliders.

The work of R.N.M. was supported by the U.S. National Science Foundation under Grant No. PHY1620074. Y.Z. is grateful to the Center for High Energy Physics, Peking University for the hospitality, the local support, and the active discussions during the visit.

[1] C. Patrignani et al. (Particle Data Group), Chin. Phys. C 40, 100001 (2016); and 2017 update.

[2] A. de Gouvea and P. Vogel, Prog. Part. Nucl. Phys. 71, 75 (2013); H. K. Dreiner, K. Nickel, F. Staub, and A. Vicente, Phys. Rev. D 86, 015003 (2012); A. Vicente, Adv. High Energy Phys. 2015, 1 (2015); M. Raidal et al., Eur. Phys. J. C 57, 13 (2008).

[3] M. Lindner, M. Platscher, and F. S. Queiroz, Phys. Rep. 731, 1 (2018).

[4] C. S. Aulakh and R. N. Mohapatra, Phys. Lett. 119B, 136 (1982); L. J. Hall and M. Suzuki, Nucl. Phys. B231, 419 (1984); G. G. Ross and J. W. F. Valle, Phys. Lett. 151B, 375 (1985); R. Barbier et al., Phys. Rep. 420, 1 (2005);

[5] P. S. B. Dev, R. N. Mohapatra, and Y. Zhang, J. High Energy Phys. 05 (2016) 174; Phys. Rev. D 95, 115001 (2017); Nucl. Phys. B923, 179 (2017); A. Maiezza, G. Senjanović, and J. C. Vasquez, Phys. Rev. D 95, 095004 (2017).

[6] P. Q. Hung, Phys. Lett. B 649, 275 (2007); 659, 585 (2008); J. P. Bu, Y. Liao, and J. Y. Liu, Phys. Lett. B 665, 39 (2008); C. F. Chang, C. H. V. Chang, C. S. Nugroho, and T. C. Yuan,
Nucl. Phys. B910, 293 (2016); P. Q. Hung, T. Le, V. Q. Tran, and T. C. Yuan, arXiv:1701.01761.

[7] G. C. Branco, P. M. Ferreira, L. Lavoura, M. N. Rebelo, M. Sher, and J. P. Silva, Phys. Rep. 516, 1 (2012); A. Crivellin, J. Heeck, and P. Stoffer, Phys. Rev. Lett. 116, 081801 (2016).

[8] See Supplemental Material at http://link.aps.org/ supplemental/10.1103/PhysRevLett.120.221804 for more details of the model frameworks and experimental constraints of the LFV couplings, which includes Refs. [9-14].

[9] J. Kersten and A. Y. Smirnov, Phys. Rev. D 76, 073005 (2007).

[10] C. H. Lee, P. S. B. Dev, and R. N. Mohapatra, Phys. Rev. D 88, 093010 (2013).

[11] T. E. Clark and S. T. Love, Mod. Phys. Lett. A 19, 297 (2004).

[12] U. D. Jentschura, G. Soff, V. G. Ivanov, and S. G. Karshenboim, Phys. Rev. A 56, 4483 (1997); U. D. Jentschura, S. G. Karshenboim, V. G. Ivanov, and G. Soff, Phys. Lett. B 424, 397 (1998); I. F. Ginzburg, U. D. Jentschura, S. G. Karshenboim, F. Krauss, V. G. Serbo, and G. Soff, Phys. Rev. C 58, 3565 (1998).

[13] M. Sher and Y. Yuan, Phys. Rev. D 44, 1461 (1991).

[14] R. Harnik, J. Kopp, and J. Zupan, J. High Energy Phys. 03 (2013) 026.

[15] CEPC-SPPC Study Group, Reports No. IHEP-CEPCDR-2015-01, No. IHEP-TH-2015-01, and No. IHEP-EP2015-01.

[16] H. Baer et al., arXiv:1306.6352.

[17] M. Bicer et al. (TLEP Design Study Working Group), J. High Energy Phys. 01 (2014) 164.

[18] E. Accomando et al. (CLIC Physics Working Group), arXiv: hep-ph/0412251.

[19] V. V. Kabachenko and Y. F. Pirogov, Eur. Phys. J. C 4, 525 (1998); G.-C. Cho and H. Shimo, Mod. Phys. Lett. A 32, 1750127 (2017).

[20] P. M. Ferreira, R. B. Guedes, and R. Santos, Phys. Rev. D 75, 055015 (2007); J. I. Aranda, A. Flores-Tlalpa, F. Ramirez-Zavaleta, F. J. Tlachino, J. J. Toscano, and E. S. Tututi, Phys. Rev. D 79, 093009 (2009); B. Murakami and T. M. P. Tait, Phys. Rev. D 91, 015002 (2015).

[21] S. Banerjee, B. Bhattacherjee, M. Mitra, and M. Spannowsky, J. High Energy Phys. 07 (2016) 059; I. Chakraborty, A. Datta, and A. Kundu, J. Phys. G 43, 125001 (2016); I. Chakraborty, S. Mondal, and B. Mukhopadhyaya, Phys. Rev. D 96, 115020 (2017); Q. Qin, Q. Li, C. D. Lü, F. S. Yu, and S. H. Zhou, arXiv:1711.07243.

[22] C. Hays, M. Mitra, M. Spannowsky, and P. Waite, J. High Energy Phys. 05 (2017) 014.

[23] W. Rodejohann and H. Zhang, Phys. Rev. D 83, 073005 (2011); T. Nomura, H. Okada, and H. Yokoya, Nucl. Phys. B929, 193 (2018).

[24] L. Calibbi and G. Signorelli, Riv. Nuovo Cimento 41, 71 (2018)

[25] L. Willmann et al., Phys. Rev. Lett. 82, 49 (1999).

[26] P. J. Mohr, D. B. Newell, and B. N. Taylor, Rev. Mod. Phys. 88, 035009 (2016).

[27] W. S. Hou and G. G. Wong, Phys. Rev. D 53, 1537 (1996).

[28] J. Abdallah et al. (DELPHI Collaboration), Eur. Phys. J. C 45, 589 (2006). 
[29] A. Belyaev, N. D. Christensen, and A. Pukhov, Comput. Phys. Commun. 184, 1729 (2013).

[30] C. Milstene, G. Fisk, and A. Para, J. Instrum. 1, P10003 (2006); D. Yu, M. Ruan, V. Boudry, and H. Videau, Eur. Phys. J. C 77, 591 (2017).
[31] A. Hammad, S. Khalil, and C. S. Un, Phys. Rev. D 95, 055028 (2017).

[32] L. Bian, J. Shu, and Y. Zhang, J. High Energy Phys. 09 (2015) 206. 